\title{
Intelligent Technique for Signal Processing to Identify the Brain Disorder for Epilepsy Captures Using Fuzzy Systems
}

\author{
Sasikumar Gurumurthy ${ }^{\mathrm{a}}$, B.K.Tripathy \\ School of Computing Science and Engineering, VIT University, India
}

\begin{abstract}
The new direction of understand the signal that is created from the brain organization is one of the main chores in the brain signal processing. Amid all the neurological disorders the human brain epilepsy is measured as one of the extreme prevalent and then programmed artificial intelligence detection technique is an essential due to the crooked and unpredictable nature of happening of epileptic seizures. We proposed an Improved Fuzzy firefly algorithm, which would enhance the classification of the brain signal efficiently with minimum iteration. An important bunching technique created on fuzzy logic is the Fuzzy C means. Together in the feature domain with the spatial domain the features gained after multichannel EEG signals remained combined by means of fuzzy algorithms. And for better precision segmentation process the firefly algorithm is applied to optimize the Fuzzy C-means membership function. Simultaneously for the efficient clustering method the convergence criteria are set. On the whole the proposed technique yields more accurate results and that gives an edge over other techniques. This proposed algorithm result compared with other algorithms like fuzzy c means algorithm and PSO algorithm.
\end{abstract}

\section{Introduction}

Epilepsy captures is considered as the maximum collective neurological disorder that disturbs $1-3 \%$ of the world's inhabitants. This one is considered through the amount of two or supplementary meaningless epileptic captures that remain irregular rhythmic exoneration of electrical movement of the brain. This spectrum of brain disorders ranges from severe, life-threatening and disabling, to ones that are much more benign. In epilepsy, the normal pattern of neuronal activity becomes disturbed, causing strange sensations, emotions and behaviour or sometimes convulsions, muscle spasms and loss of consciousness. [1] There are many possible causes of epilepsy with several types of seizures. Anything that disrupts the normal pattern of neuron activity (from illness to brain damage to abnormal brain development) can lead to seizures. Epilepsy may develop because of an anomaly in brain wiring, an unevenness of nerve signalling chemicals called neurotransmitters, changes in the vital features of brain cells called channels or a combination of these factors and other possible factors. Having a single seizure as the result of a high fever (called febrile seizure) Common diagnostic tests for epilepsy are measurement of electrical activity in the brain and brain scans such as magnetic resonance imaging (MRI) or computed tomography (CT).

Paroxysmal alteration of single or extra neurological occupations such as motor, behaviour and autonomic occupations is definite as a seizure. Epileptic seizures remain episodic, quickly developing fleeting actions, normally enduring for less than a minute. There are numerous studies to understand the machine after epileptic capture and however it is not entirely known yet, a seizure event can be developed as the enlarged network excitation of the neural networks with synchronous discharge as well as variable propagation in the brain. In crucial epilepsy, a specific brain region may candidate for the epilepsy event, but in comprehensive epilepsy the complete brain might remain candidate aimed at seizure events. The utmost broadly castoff quantity aimed at analysis of neurological syndromes such as epilepsy in medical backgrounds is electroencephalogram (EEG). Enduring intensive care of EEG is one of the best skilled conducts aimed at analysis of epilepsy. This one delivers evidence almost forms of brain electrical movement, category then occurrence of captures and seizure emphasis laterality. In abiding monitoring, ictal EEG footage is typically connected through the medical appearance of removal. One of the greatest defining features in seizure recognition before primary recognition is the assignment of electrodes [8]. Unknown footage location is where the capture attention is placed; the variations in EEG can happen earlier the medical appearances. But if the electrodes are located in remote place after the seizure beginning spot, the medical appearances could transpire earlier any graphic variations in EEG [4]. Professionals observing abiding EEG footages typically look on behalf of initial visually specious variations in EEG to classify ictal arrival.

\footnotetext{
${ }^{\mathrm{a}}$ sasichief@gmail.com
} 


\section{Related Work}

Epilepsy is one of the neurological disorders under extensive research and over the years there have been several methods of seizure detection. One of method was based on detecting strong rhythmic movements of the patient. The drawback in these methods is that, seizures do not always present strong movements. This setback led the detection problem to apply methods based on EEG signal analysis, for example, J.R. Ives \& Woods (1974) described detection of large seizures discharges in several EEG channels by amplitude discrimination; T.L. Babb \& Crandall (1974) designed an electronic circuit for seizures detection from intracranial electrodes [9].

\subsection{Feature Extraction}

Finding a set of measurements or a block of information with the aim of expounding in a well-defined way the data or an event present in a signal is called feature extraction. For detection, a classification or regression task in biomedical signal processing these measurements or features is the fundamental basis and is one of the key steps in the data analysis process [7]. These features constitute a new form of expressing the data, and can be binary, categorical or continuous, and also represent attributes or direct measurements of the signal. For example, features may be age, health status of the patient, family history, electrode position or EEG signal descriptors (amplitude, voltage, phase, frequency, etc.) [8]. More formally, feature extraction assumes we have for $\mathrm{N}$ samples and $\mathrm{D}$ features, a matrix $\mathrm{N} \times \mathrm{D}$, where $\mathrm{D}$ represents the dimension of the feature matrix. That means, at the sample $\mathrm{n}$ from the feature matrix, we could obtain an unidimensional vector $\mathrm{x}=[\mathrm{x} 1, \mathrm{x} 2, \ldots, \mathrm{xD}]$ called as "pattern vector." Guyon et al. (2006) consists several methods in EEG feature extraction [10].

The Time domain, frequency domain features, and entropy-based features were extracted from EEG segments. The four features used in this study were average amplitude, rhythmicity (coefficient of variation of amplitude), dominant frequency, and entropy. These features are known to contain the most discriminant information for detecting seizure events. Features extraction methods are expounded briefly in the following sections.

\subsection{Average Amplitude}

For time-based development of limited seizures, average amplitude (AVA) is a decent amount. EEG signals display rhythmic movement with a recurrence frequency among 3 and $30 \mathrm{~Hz}$ throughout limited seizures. Consequently, to calculate regular amplitude, EEG divisions were initially high-pass cleaned overhead $3 \mathrm{~Hz}$ to eliminate low-frequency sound. Then to identify peaks, a peak recognition algorithm created on the zerocrossings of the first derivative of EEG signals was used. Then, by taking the average of the amplitudes of their half waves the amplitudes of the peaks were computed.
Lastly, the average amplitude () was calculated by taking the average of the amplitudes of the identified peaks [1].

\subsection{Rhythmicity}

Coefficient of variation of amplitude (CVA) is a measure of rhythmicity or regularities of ictal activities. During seizure evolution, the regularity of the amplitude of EEG tends to increase slowly; this increase is characterized by the CVA. In case of partial seizures, the signals exhibit strong rhythmic characteristics that are likely to have regularity in amplitude. The coefficient of variation (CVA) quantifies the increased regularity observed during partial seizures. The CVA is defined as the ratio of the standard deviation of absolute amplitude to the mean absolute amplitude as where is the standard deviation and is the mean of each EEG segment [1].

\subsection{Entropy}

Entropy was initially introduced by Shannon in 1948 and is briefly defined as a measure of "irregularity" or "uncertainty". The Shannon entropy () is computed as where are the probabilities of a datum in bin. Pincus and Goldberger introduced approximate entropy (ApEn) are considered more appropriate to compute the entropy for short and noisy time series data. A low value of the entropy indicates that the time series is deterministic, whereas a high value indicates randomness. Therefore, a high value of entropy indicates the irregularities in the EEG data. To compute ApEn, it is required to determine a run length and a tolerance window to measure the likelihood between runs of patterns [3]. The tolerance window and embedding dimension are the two important parameters in computation of ApEn. In this study, Sample Entropy (SampEn), which is a variant of approximate entropy to quantify entropy of EEG, was used considering its robustness over ApEn. Sample Entropy is the negative natural logarithm of an estimate of the conditional probability that segments of length that match point wise within a tolerance also match at the next point. Therefore, for investigating dynamics of biomedical signal and other time series this measure is a useful tool [1].

\subsection{Domain Frequency}

The peak with the maximum spectral power in the power spectrum of a signal is defined as dominant frequency $\mathrm{O}$. This feature is particularly important in distinguishing ictal activities from interictal activities by quantizing the frequency signature information mostly found in partial seizures. This is characterized by a high-frequency activity at seizure onset and a low-frequency activity at the end of the seizures [9]. In this study, parametric spectrum estimation method, autoregressive-modeling (AR) approach, was used to estimate the spectral frequency band of the short EEG segments. The AR model order was chosen according to Akaike information criterion. The Burg method was used for computing the 
AR coefficients for short EEG segments. Then, the spectral power of a given segment is estimated using these AR coefficients. For every spectral peak, the spectral frequency band was defined as the frequencies at rising and falling slopes of the peak with half the amplitude of the peak.

\section{Fuzzy C-means clustering}

Fuzzy c-means (FCM) is a method of clustering which allows a [13] datum to belong to more than one cluster by varying degree of membership described by a fuzzy matrix with $\mathrm{n}$ rows and c columns in which $\mathrm{n}$ is the number of data and $\mathrm{c}$ is the number of clusters: Fuzzy cmeans algorithm is popularly used clustering algorithm and introduced by Bezek in 1974, FCM algorithm result totally depend on the initialization of center, the random selection of the center causes the local optima problem. To solve the shortcoming of the FCM algorithm, this proposal integrate the optimization algorithm with FCM [11]

Consider the set of vectors $(X=(x 1, x 2, \ldots . x n) 2<=c<=n)$ clustering into $\mathrm{c}$ groups. The set of fuzzy partition matrices is denoted as

$\mathrm{M}_{\mathrm{fc}}=\left\{W \in R^{c n}\left|W_{i R} \in[0,1], \forall i, K_{i} \Sigma_{l=1} W_{i k}=1, \forall K\right|\right.$

$$
0<\sum_{n=1} w_{k} \propto n \forall t
$$

where $1<=\mathrm{i}<=\mathrm{c}, 1<=\mathrm{k}<=\mathrm{n}$

FCM objective function computed by using following equation and Euclidian distance [11]

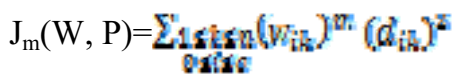

$$
\begin{aligned}
& \left\{d_{k_{k}}\right\rangle=\left\|x_{k_{k}}-p_{l}\right\|
\end{aligned}
$$

\section{Firefly Algorithm}

First proposed by Yang, a Firefly Algorithm (FA) is an optimization algorithm, which simulates the flash pattern and features of fireflies. The Firefly Algorithm is a population-based algorithm to discover the global optima of objective functions based on swarm intelligence. Individually firefly is enticed by the brighter luminosity of additional adjacent fireflies. The attractiveness decreases when far between the couples of fireflies [14].

Three idealized rules in firefly algorithm distinct by Yang:

1) All fireflies are unisex so that one firefly will be attracted to other firefly irrespective of their sex;

2) Attractiveness is proportional to their brightness. Thus, for any two fireflies, the less bright one will move towards the brighter one. If there is no brighter one than a particular firefly, it will move randomly;

3) The brightness of a firefly is from the objective function. For a maximization problem, the brightness can simply be proportional to the value of the objective function.

X-S Yang made use of firefly algorithm like an optimization algorithm for different purposes .In the design of real-world engineering problems, Azad and Azad and Gandomi showed that firefly algorithm shall be a good remedy for multimodal optimization problems. Banati and Bajaj showed that FA should be a potential future prospective for efficient performance in terms of time and optimality for feature selection when compared with other algorithms [11]. Horng qualified that fireflybased method (firefly-based algorithm) illustrated better high speed and good reconstructed images while compressing the digital images.

The attractiveness function $\beta$ calculate from the distance $r(i, j)$ of the firefly is determined by:

$$
f(x) f)=\beta 0 \varepsilon^{-\gamma t h r^{2}}
$$

Where, is the attractiveness at and is the light absorption coefficient at the source. It should be noted that the which is the euclidence distance between any two fireflies and at and, where and are the spatial coordinate of the fireflies and, respectively.

The movement of a firefly $i$,which is attracted to another more attractive firefly $j$, is decided by:

$$
\left.\beta\left(w_{1}\right)\right)_{l}=x_{l}+\beta 00^{-w h / 2}\left(x_{l}-x_{j}\right)+\alpha\left(\operatorname{rand}-\frac{1}{3}\right)
$$

$\alpha$ is the randomization parameter, in most of cases $\alpha$ between $[0,1], \beta 0=1$ and $\gamma e[0,01,100]$.

In various signal processing and pattern recognition applications fuzzy logic has been extensively used. Fuzzy rules can be defined using experts' knowledge for decision making which are simpler to implement and modular as well. Increasing the number of rules one can increase the accuracy of the model. With less complex mathematical analysis and modelling, processing speed can also be significantly improved. More over fuzzy logic is a useful method for non- linear input-output mapping which is effective in seizure detection or early detection applications. Artificial neural networks and support vector machines are other prominent detection methods which demands training, complex mathematical analysis, and modelling. All these major setbacks can be avoided in fuzzy logic. Therefore, in this study, we applied adaptive version of fuzzy logic system with a unique approach of merging information in feature as well as spatial and temporal domain.

\section{Fuzzy Firefly Algorithm}

Fuzzy-based Firefly algorithm (FFA) for data clustering is proposed. Each solution of the cluster is defined fuzzily and then the solution is updated using firefly algorithm 
the same way it does with non-fuzzy solution. However, minimization of the objective function is calculated in the same way as FCM algorithm.in the proposed method cluster centers are the decision parameters to minimize the membership function. A single firefly represents the cluster centers vectors. Eq. 5 represents each firefly

$X_{i}=\left(p_{i l}, \ldots ., p_{i j}, \ldots, p_{i c}\right), 2 \leq j \leq c$

Where $f_{i j}$ represents the jth center vector. $n$ number of fireflies represents $\mathrm{n}$ candidates solutions. This is a minimize problem the intensity of each firefly is equal to the values of the objective function of FCM.FCM will terminate when there is no scope for further improvement in the member function. FFA will terminate when the no changes in the 5 consecutive iterations. Improved FFA method has 50 is the maximum iteration or no changes in current best in 3 consecutive iterations.

Pseudo code of the algorithm:

\section{Initialize the population of $\boldsymbol{n}$ fireflies with $\boldsymbol{C}$ random cluster centers of d-dimensions \\ Initialize the algorithm parameters \\ Repeat:}

\section{For $\boldsymbol{i}=1: n$ all $\boldsymbol{n}$ fireflies \\ For $j=1: n$ all $n$ fireflies \\ Calculate light intensity (membership function value) of each firefly

$$
\text { If }\left(I_{i}>I_{j}\right)
$$ fireflies \\ Move firefly I towards $j$ to update the position of}

\section{Rank the fireflies and find current best one \\ Until: maximum iteration or minimum changes in the member function}

\section{Results and Discussion}

Our experimental study is to compare the proposed algorithm with other popular optimization algorithms like PSO, FFA, and FCM. The dataset collected from the "ictal" information records from Freiburg project, which must captures with at least 50 minutes of preictal data and postictal data with no specified duration. Consequently, the false recognition rate per hour is slight complex associating to additional approaches in the works but sensible seeing the assessment dataset.

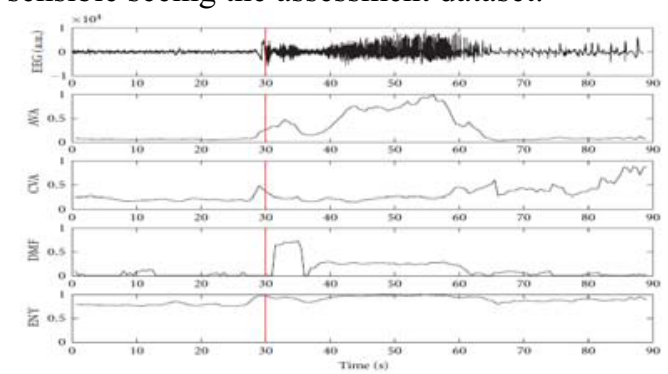

Figure 1. Seizure is marked by red line. Corresponding changes in features also shown. (a) Top subplot: an example of a seizure evolution in iEEG. (b) Bottom four subplots: corresponding changes in characteristics features: Average amplitude (AVA), coefficient of variation of amplitude (CVA), dominant frequency (DMF), and entropy (ENY). Seizure onset is marked by red vertical line. Early electrographic changes are visual in three of the four features.

For each of the patients, there are datasets called "ictal" and "interictal", the former containing files with epileptic seizures and at least 50 min pre-ictal data [10].

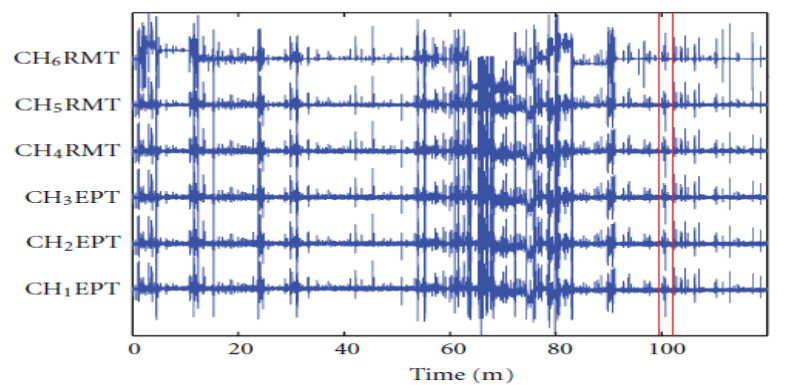

Figure 2. Seizure evolution profile in iEEG obtained from patient 10. Seizure onset and offset times are marked by red vertical lines, respectively. Acronyms: CH1EPT: Epileptic channel 1, CH4RMT: Remote channel 4.

Therefore, the false detection rate per hour is little higher comparing to other methods in the literature but reasonable considering the evaluation dataset. Till date, many algorithms for epilepsy and seizure detection have been developed with different degrees of success [2-15]. Here, we have discussed briefly some of these methods providing a scope of comparison with our method. In a recent study, Zhang et al. Proposed an automatic patientspecific method for seizure onset detection using a novel incremental learning scheme based on nonlinear dimensionality reduction. Feature sets were extracted using continuous wavelet transform (CWT). Considering computation time and resources, the choice of discrete wavelet transform might have been better. Their method was evaluated on iEEG recordings from 21 patients obtained from Freiburg project with duration of 193.8 hour and 82 seizures.

In this paper, different thresholds were used for different patients and a postprocessor was utilized to reduce the false detections in two steps. First short length detections (less than $5 \mathrm{~s}$ ) and artifacts were rejected. Secondly, two consecutive detections were unified given that they are less than a predefined minimum time interval (set to $30 \mathrm{~s}$ ). Chan et al. presented a novel patient-specific algorithm for seizure onset detection and accurate onset time determination. The algorithm extracts spectral and temporal features in five frequency bands within a sliding window and the feature windows were classified as containing or not containing a seizure onset using support vector machines SVMs). Support vector machine is a popular classification paradigm for epileptic seizure detection and prediction being used by many researchers in this area. In order to accurately localize the seizure onsets in time, the method makes use of clustering and regression analysis. Therefore, their algorithm yielded precise detection in time as reported in five of the six patients, at least $90 \%$ of the latencies are less than $3 \mathrm{~s}$ resulting median detection latency less than $100 \mathrm{~ms}$ with 
standard deviation less than 3 s. However, the method utilizing user-adjustable parameters allow tuning to achieve high detection sensitivity, low false positive rate, and low detection latencies. Standard cross-validation performance measures resulted sensitivities in the range of $80 \%$ to $98 \%$ and false positive rates from 0.12 to $2.8 / \mathrm{h}$ [6]. Gardner et al. [5] presented a detection latency which is negative in time $(-7.58 \mathrm{~s})$ however with a higher false detection rate of 1.56 false detections per hour.

\subsection{Sensitivity}

Sensitivity is significant quantity for event based system performance assessment. It measures the capability of a system to identify seizure suitably.

It is the amount of true positive rate and distinct as the ratio of the number of correctly detected seizure to the total number of seizures.

Sensitivity $=(\mathrm{TP} /(\mathrm{TP}+\mathrm{FN})) \times 100(6)$

True Positive (TP): The system detects the actual seizures

False Negative (FN): The System misses the actual seizures

Table 1. Show the comparison sensitivity and detection latency for various algorithms.

\begin{tabular}{|l|c|c|c|}
\hline \multicolumn{1}{|c|}{ Algorithm } & Sensitivity & $\begin{array}{c}\text { Average } \\
\text { detection } \\
\text { latency (s) }\end{array}$ & $\begin{array}{c}\text { FDR } \\
/ \mathbf{h}\end{array}$ \\
\hline FCM & 94.5 & 29 & 0.35 \\
\hline Fuzzy+ PSO & 91.3 & 35 & 0.32 \\
\hline FFA & 94.7 & 28 & 0.28 \\
\hline Improved FFA & 96.8 & 13.5 & 0.23 \\
\hline
\end{tabular}

Table 1: comparison of objective function value and no of iteration for the dataset for various algorithms. When compare to the other algorithm Improved version of FFA detect the seizure with minimum iterations.

Our method achieved average sensitivity of $96.8 \%$ with $0.23 / \mathrm{h}$ false detection rates. The average detection latency achieved was 13.5 seconds. Firefly algorithm minimizes the detection latency of the classification process.

\section{Conclusions and Future Work}

After extensive research, in this paper we existing a strong technique of classifying seizure using fuzzy firefly algorithm classification. Firefly algorithm helps the fuzzy system to obtain the better classification in minimum iteration. This proposed algorithm converge the result faster than the other algorithm with minimum iterations. Considering the challenge and important development in the capacity of seizure recognition, we primarily absorbed in designing effective seizure recognition classification with low complexity. In future our work will compare with other various classification algorithm like SVM and optimization algorithm like Genetic algorithm.

\section{References}

1. Alessandro, D.M, R. Esteller, G. Vachtsevanos, A. Hinson, J. Echauz, and B. Litt (2003). Epileptic Seizure Prediction Using Hybrid Feature Selection over Multiple Intracranial EEG Electrode Contacts: A Report of Four Patients. IEEE Transaction on Biomedical Engineering. Vol.50, No.5. pp. 603-615.

2. Carlos, G. M, A.M. Trigueros, A. N. Vazquez (2012). EEG Signal Processing for Epilepsy. EEG Signal Processing for Epilepsy. Histological, Electroencephalographic and Psychological Aspects, Dr. Dejan Stevanovic (Ed.). pp. 1-27.

3. EEG database: seizure Prediction project Freilburg http://epilepsy.uni-freiburg.de/freiburg-seizureprediction-project/eeg-database

4. Izakian, .H and A. Abraham (2011). Fuzzy C-means and Fuzzy swarm clustering Problem. Expert System with Application. Vol.38, No.3. pp. 1835- 38.

5. Jitpakdee, P., and P. Aimmanee (2014). Image Cluster using fuzzy based firefly algorithm. Parisut ICICTES2014, Thailand. Vol.1, No.2. pp. 1-8.

6. Lotte, F., M. Congedo, A. Lécuyer, F. Lamarche and B. Arnaldi (2007). A review of classification algorithm for EEG-based brain-computer interfaces. Journal of Neural Engineering. Vol.4, No.2, pp.1-23.

7. Nandy, S., P. P. Sarkar, A. Das (2012). Analysis of a Nature Inspired Firefly-Algorithm Based Back Propagation Neural Network Training. International Journal of Computer Applications. Vol.43 (22). pp. 816.

8. Rabbi, .F and R.F. Rezai (2012). Fuzzy Logic System for Seizure Onset Detection in Intracranial EEG, Computational Intelligence and NeuroScience. Hindawi Publication Corporation. Vol. 2012, Article.ID.705140. pp. 1-12.

9. Runkler, T.A., and C. Katz (2006). Fuzzy clustering by Particle Swarm Optimization. Proceedings of 2006, IEEE international Conference on Fuzzy Systems, Canada. pp. 601-608.

10. Senthilnath, J., S.N. Omkar, V. Mani (2011). Clustering using Firefly algorithm: Performance Study. Swarm evolutionary computation. Vol.1, No.3. pp. 164-171.

11. Saibal K.P, C.S.Rai, A.P. Singh (2012). Comparative Study of Firefly Algorithm and Particle Swarm Optimization for Noisy Non Linear Optimization Problems. International Journal of Intelligent Systems and Applications. Vol.4, No.10. pp. 50-57.

12. Yang, X. S. (2010). Firefly algorithm Stochastic Test Functions and Design Optimization. International Journal of Bio-Inspired Computation. Vol.2, No.4. pp. 78-84.

13. Yang, X. S. (2008). Nature-inspired metaheuristic Algorithm. Luniver Press. pp. 741-751. 